\title{
Clinical application of the AUC-guided
} dosage adjustment of docetaxel-based chemotherapy for patients with solid tumours: a single centre, prospective and randomised control study

Ning Sun ${ }^{1 \dagger}$, Bo Shen ${ }^{1 \dagger}$, Jiali Zhu ${ }^{1}$, Xiaomei Zhang ${ }^{1}$, Huayun Zhu', Geyu Liang ${ }^{2}$, Deliang Yang ${ }^{1}$, Jianwei Lu ${ }^{1}$ and Yan Zhang ${ }^{1 *}$

\begin{abstract}
Background: Docetaxel (DTX) is a widely used anti-tumour drug, and its dosage is solely determined by body surface area (BSA). Adverse events, such as neutropenia or unsatisfied efficacy, likely occur because of differences in the pharmacokinetics (PK) and pharmacodynamics of patients. Thus, a feasible dosage adjustment method is needed.

Methods: A total of 209 eligible patients who provided consent were enrolled and randomised into two groups to receive the BSA- and PK-guided dosage adjustments of DTX-based chemotherapy (3 weeks per cycle). The AUC of DTX was detected, and the therapeutic window for Chinese patients was determined. The proportion of patients within the therapeutic window was evaluated. Neutropenia was examined in accordance with the toxicity grading standard suggested by the World Health Organisation. Tumour response was assessed in accordance with Response Evaluation Criteria in Solid Tumors version 1.1. The primary endpoint was the incidence of neutropenia, and the secondary endpoints were disease control rate (DCR) and 3-year survival rate.

Results: The therapeutic window for Chinese patients was $1.7-2.5 \mathrm{mg} \cdot \mathrm{h} / \mathrm{L}$. The proportion of patients within the therapeutic window was $63.89 \%$ versus $28.33 \%(P<0.0001)$, and the incidence of neutropenia was $68.33 \%$ versus $38.89 \%(P=0.001)$ in the experimental group versus the control group in the sixth cycle, respectively. DCR was $72 \%$ versus $85 \%(P=0.018)$ in the control group versus the experimental group. The 3 -year survival rate of the PK group was significantly higher than that of the BSA group $(P=0.034)$.
\end{abstract}

Conclusions: The PK-guided dosage adjustment of DTX could significantly increase the proportion of patients within the therapeutic window, decrease the incidence of neutropenia and increase the DCR and the 3-year survival rate. The PK-guided dosage adjustment based on the dynamic monitoring of AUC could be a useful method for oncologists to improve individualised treatment options, optimise drug efficacy and reduce drug toxicity.

\footnotetext{
*Correspondence: zhangyanonco@outlook.com

${ }^{\dagger}$ Ning Sun and Bo Shen contributed equally to this work

${ }^{1}$ Department of Medical Oncology, Jiangsu Cancer Hospital \& Jiangsu

Institute of Cancer Research \& The Affiliated Cancer Hospital of Nanjing

Medical University, No. 42, Baiziting, Nanjing 210009, China

Full list of author information is available at the end of the article
}

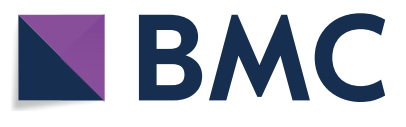

(c) The Author(s) 2020. This article is licensed under a Creative Commons Attribution 4.0 International License, which permits use, sharing, adaptation, distribution and reproduction in any medium or format, as long as you give appropriate credit to the original author(s) and the source, provide a link to the Creative Commons licence, and indicate if changes were made. The images or other third party material in this article are included in the article's Creative Commons licence, unless indicated otherwise in a credit line to the material. If material is not included in the article's Creative Commons licence and your intended use is not permitted by statutory regulation or exceeds the permitted use, you will need to obtain permission directly from the copyright holder. To view a copy of this licence, visit http://creativeco mmons.org/licenses/by/4.0/. The Creative Commons Public Domain Dedication waiver (http://creativecommons.org/publicdomain/ zero/1.0/) applies to the data made available in this article, unless otherwise stated in a credit line to the data. 
Keywords: Docetaxel, Body surface area, Pharmacokinetics, Dosage

\section{Background}

Docetaxel (DTX) is a paclitaxel-based antineoplastic drug that plays an antineoplastic role by interfering with the microtubule network necessary for cell mitosis and mitotic interphase [1]. DTX can bind to free tubulin, promote tubulin assembly into stable microtubules and inhibit its depolymerisation; this mechanism of action results in microtubule bundle malfunction and microtubule immobilisation, thereby inhibiting cellular mitosis [2]. DTX is widely used in the treatment of breast cancer, ovarian cancer, non-small cell lung cancer, gastric cancer, pancreatic cancer, melanoma and other cancer types [3, 4].

Similar to the dosage of other chemotherapeutic reagents, DTX dosage is calculated solely on the basis of a patient's body surface area (BSA). However, clinical data suggest that administering patients with the same dosage of DTX results in significant pharmacokinetic (PK) differences because of internal or external factors, such as genomic composition, physiological status, genetic characteristics and living habits [5]. Remarkable individual pharmacodynamic differences are also observed because of the narrow effective therapeutic range (therapeutic window) of DTX blood concentration [6]. A low drug concentration may elicit unsatisfactory therapeutic effects, whereas a high drug concentration likely leads to increased toxicity and adverse effects, which are the main reasons for the high toxicity of chemotherapeutic drugs and treatment failure [7]. Therefore, a feasible dosage adjustment method for each patient is needed.

Neutropenia is one of the main dose-limiting toxic effects of DTX. It can induce neutropenic fever, reduce dosage and result in early treatment discontinuation [7]. The risk factors of neutropenia include old age, low weight, high DTX dose and numerous chemotherapy cycles [8].

The area under a curve (AUC) of blood concentration is an important index for evaluating the absorption and PK of drugs in vivo. Neutropenia is closely correlated with the AUC of DTX amongst patients receiving DTX-based chemotherapy [9]. Bruno et al. [10] statistically analysed the PK data of 640 patients receiving DTXbased chemotherapy and found that AUC is statistically correlated with the incidence of neutropenia. Therefore, we hypothesised that dosage adjustment based on the dynamic monitoring of the AUC of DTX for each patient might be a useful method for oncologists to improve individualised treatment options, optimise drug efficacy and reduce drug toxicity [11].
In the present study, 209 patients who had solid tumours and received DTX-based chemotherapy were enrolled and randomised into a PK-directed DTX dosage adjustment group (PK group or experimental group) and a BSA-based dosage group (BSA group or control group). An optimised therapeutic window for the Chinese population was explored and defined, and the proportion of patients within this therapeutic window was evaluated. The objective response rate (ORR), disease control rate (DCR), 3-year survival rate and neutropenia incidence were also evaluated.

\section{Methods}

\section{Ethical statements}

The Ethical Committee of Jiangsu Cancer Hospital approved the study, and all the patients signed an informed consent for the use of their personal data for research purposes. This study was conducted in accordance with the Declaration of Helsinki, Guidelines for Good Clinical Practice and the local laws and regulations of China.

\section{Study design, patient eligibility and treatment}

This work was a single-centre, prospective, randomised controlled study.

The eligibility criteria of the study were as follows: (1) male or female aged over 18 years; (2) histologically confirmed solid tumours that could not be subjected to surgery or radiotherapy; (3) Eastern Cooperative Oncology Group score of 0-2, life expectancy of not less than 12 weeks; and (4) organ function level that met the following requirements: absolute neutrophil count $\geq 1.5 \times 10^{9} / \mathrm{L}$, platelet count $\geq 75 \times 10^{9} / \mathrm{L}$ and haemoglobin $\geq 90 \mathrm{~g} / \mathrm{L}$; serum total bilirubin of less than 1.5 times the upper limit of the normal value; aspartate aminotransferase and alanine aminotransferase of less than 2.5 times the upper limit of the normal value and serum creatinine of less than 1.5 times the upper limit of normal value; or creatinine clearance rate of more than $50 \mathrm{~mL} /$ min. The exclusion criteria were as follows: (1) received surgery or radiotherapy in the past 3 months; (2) abnormal liver, kidney, heart function and blood routine; and (3) patients with insufficient compliance in this experiment or receiving other treatments during the treatment course.

Patients who consented to the study were randomly divided into the experimental group $(n=109$, also referred to as the PK group) and the control group ( $n=100$, also referred to as BSA group). All eligible 
patients received DTX-based chemotherapy 3 weeks per cycle. Chemotherapy did not exceed six cycles. In accordance with the patient's physical condition, dosage was calculated on the basis of a BSA of less than $75 \mathrm{mg} /$ $\mathrm{m}^{2}$. The drug was administered via an intravenous drip and completed within $1 \mathrm{~h}$. From the second cycle, the PK group received dosage adjustment based on the PK parameter AUC of DTX. The BSA group continued to receive BSA-guided dosage. If the disease progressed or patients developed intolerable adverse reactions, treatment was discontinued, and patients received the best support of care.

\section{AUC calculation}

After $60 \mathrm{~min}$ of intravenous drip, $2-3 \mathrm{~mL}$ of blood was collected as samples 1 and 2, respectively, with an accurate recording of collection time. The blood samples were stored in a refrigerator at $2{ }^{\circ} \mathrm{C}-8{ }^{\circ} \mathrm{C}$ within $10 \mathrm{~min}$. Plasma was isolated through centrifugation within $12 \mathrm{~h}$. The blood concentration of DTX was detected using a DTX assay kit (Jiangsu Changxing Medical Technology Co., Ltd.). AUC was calculated on the basis of DTX dosage, start and end time of intravenous drip, blood sample collection time and DTX blood concentration by using the population PK model software provided by Saladax Company.

\section{Clinical assessments}

Efficacy was evaluated every two chemotherapy cycles. Response was assessed in accordance with the Response Evaluation Criteria in Solid Tumors (RECIST 1.1). DTXrelated neutrophils were classified in accordance with the toxic grading standard suggested by WHO.

\section{Outcomes and endpoints}

This study was designed to evaluate the effects of AUCbased dosage adjustment on clinical outcomes, such as the population rate within the therapeutic window and the incidence rate of neutropenia. The primary endpoint was the incidence rate of grades 3 and 4 neutropenia. The secondary endpoint was disease control rate (DCR). ORR was defined as the percentage of patients with a complete response (CR) or a partial response (PR). DCR was defined as the percentage of patients with a $\mathrm{CR}$, a PR or a stable disease (SD).

\section{Statistical methods and sample size calculation}

Data were analysed with SPSS 20.0, and measurements were expressed as mean ( \pm standard deviation, SD). $\mathrm{P}<0.05$ indicated significant differences (", $\mathrm{P}<0.05$; **, $\mathrm{P}<0.01$; ***; $\mathrm{P}<0.001)$. The incidence rate of neutropenia between the two groups was compared via a Chi square test. DCR was compared via a Mantel-Haenszel test, and survival was examined with Kaplan-Meier analysis.

\section{Results}

\section{Patient characteristics}

Patients diagnosed with solid tumours and scheduled to receive DTX-based chemotherapy in our hospital from January 2015 to December 2016 were screened, and 209 patients were enrolled in this study. The patient baseline characteristics are summarised in Table 1. The 209 eligible patients who consented to participate (59 cases of non-small cell lung cancer, 9 cases of nasopharyngeal carcinoma, 42 cases of breast cancer, 34 cases of oesophageal cancer, 42 cases of prostate cancer and 23 cases of gastric cancer) were randomised into PK $(n=109)$ and BSA $(n=100)$ groups. Of the 209 randomised patients, $130(62 \%)$ were male and $79(38 \%)$ were female. The patients were $20-84$ years old $(57 \pm 12)$. The baseline characteristics were similar between experimental and control groups and had no significant differences. All patients received treatment as allocated.

\section{AUC of DTX amongst all patients}

In the 100 patients in the BSA group, the AUC of DTX was $0.5-5.3(1.96 \pm 0.81) \mathrm{mg} \mathrm{h} / \mathrm{L}$ in the first cycle, $0.7-4.7(1.90 \pm 0.66) \mathrm{mg} \mathrm{h} / \mathrm{L}$ in the second cycle, 0.7-3.6 (1.89 \pm 0.58$) \mathrm{mg} \mathrm{h} / \mathrm{L}$ in the third cycle, 1.5$3.7(2.35 \pm 0.58) \mathrm{mg} \mathrm{h} / \mathrm{L}$ in the fourth cycle, $1.3-4.6$ $(2.68 \pm 0.72) \mathrm{mg} \mathrm{h} / \mathrm{L}$ in the fifth cycle and 1.8-4.2 $(2.85 \pm 0.59) \mathrm{mg} \mathrm{h} / \mathrm{L}$ in the sixth cycle. In the 109 patients in the PK group, the AUC of DTX was 0.5-5.1 $(1.87 \pm 0.87) \mathrm{mg} \mathrm{h} / \mathrm{L}$ in the first cycle, $0.6-3.7(1.81 \pm 0.62)$ $\mathrm{mg} \mathrm{h} / \mathrm{L}$ in the second cycle, $1.1-3.6(2.11 \pm 0.5) \mathrm{mg} \mathrm{h} / \mathrm{L}$ in the third cycle, $1.2-3.1(2.12 \pm 0.42) \mathrm{mg} \mathrm{h} / \mathrm{L}$ in the fourth cycle, $1.2-3.1(2.1 \pm 0.43) \mathrm{mg} \mathrm{h} / \mathrm{L}$ in the fifth cycle and $1.4-3.1(2.28 \pm 0.43) \mathrm{mg} \mathrm{h} / \mathrm{L}$ in the sixth cycle.

In the BSA group, the mean AUC of DTX constantly increased with the prolonged chemotherapy cycle. This result might be related to decreased tolerance and metabolism rate. The AUC was dispersed in all the cycles. In the PK group, the AUC was 0.5-5.1 $(1.87 \pm 0.87) \mathrm{mg} \mathrm{h} / \mathrm{L}$ after BSA-based dosage was administered in the first cycle, which was as dispersed as in the control group, but being less dispersed after the cycles of dosage adjustment as indicated by the decreased standard derivation (Fig. 1 and Table 2). The proportions of patients within the therapeutic window $(1.7-2.5 \mathrm{mg} \mathrm{h} / \mathrm{L})$ were $36.00 \%$ versus $36.70 \%(P=0.9166)$, $41.00 \%$ versus $50.46 \%(P=0.1705)$, $50.00 \%$ versus $61.90 \%(P=0.0928), 48.24 \%$ versus $62.64 \%$ $(P=0.0546), \quad 36.62 \%$ versus $71.08 \%(P<0.0001)$ and $28.33 \%$ versus $63.89 \%(P<0.0001)$ in the control group versus the experimental group from cycles one to six, respectively (Table 2). 
Table 1 Patient characteristics

\begin{tabular}{lcc}
\hline Variable & Experimental $(\boldsymbol{n = 1 0 9 )}$ & Control $(\boldsymbol{n}=\mathbf{1 0 0})$ \\
\hline Median age, years (SD) & $54.84(13.44)$ & $59.08(10.37)$ \\
Age group & & \\
$<65$ & $83(55)$ & $68(45)$ \\
$\geq 65$ & $26(45)$ & $32(55)$ \\
Sex & & \\
Male & $69(63)$ & $61(61)$ \\
Female & $40(37)$ & $39(39)$ \\
Type of cancer & & \\
Nasopharyngeal carcinoma & $7(78)$ & $2(22)$ \\
Lung cancer & $26(44)$ & $33(56)$ \\
Prostate cancer & $21(50)$ & $21(50)$ \\
Breast cancer & $25(60)$ & $17(40)$ \\
Oesophageal cancer & $16(47)$ & $18(53)$ \\
Gastric cancer & $14(61)$ & $9(39)$ \\
Line of therapy & & \\
1 & $62(57)$ & $52(52)$ \\
2 & $28(26)$ & $26(26)$ \\
$>2$ & $19(17)$ & $22(22)$ \\
ECOG PS & & $88(88)$ \\
$0-1$ & $93(85)$ & $12(12)$ \\
2 & $16(15)$ & 0.3101 \\
\hline Da & &
\end{tabular}

Data presented as No. (\%) unless otherwise indicated

ECOG PS Eastern Cooperative Oncology Group performance status

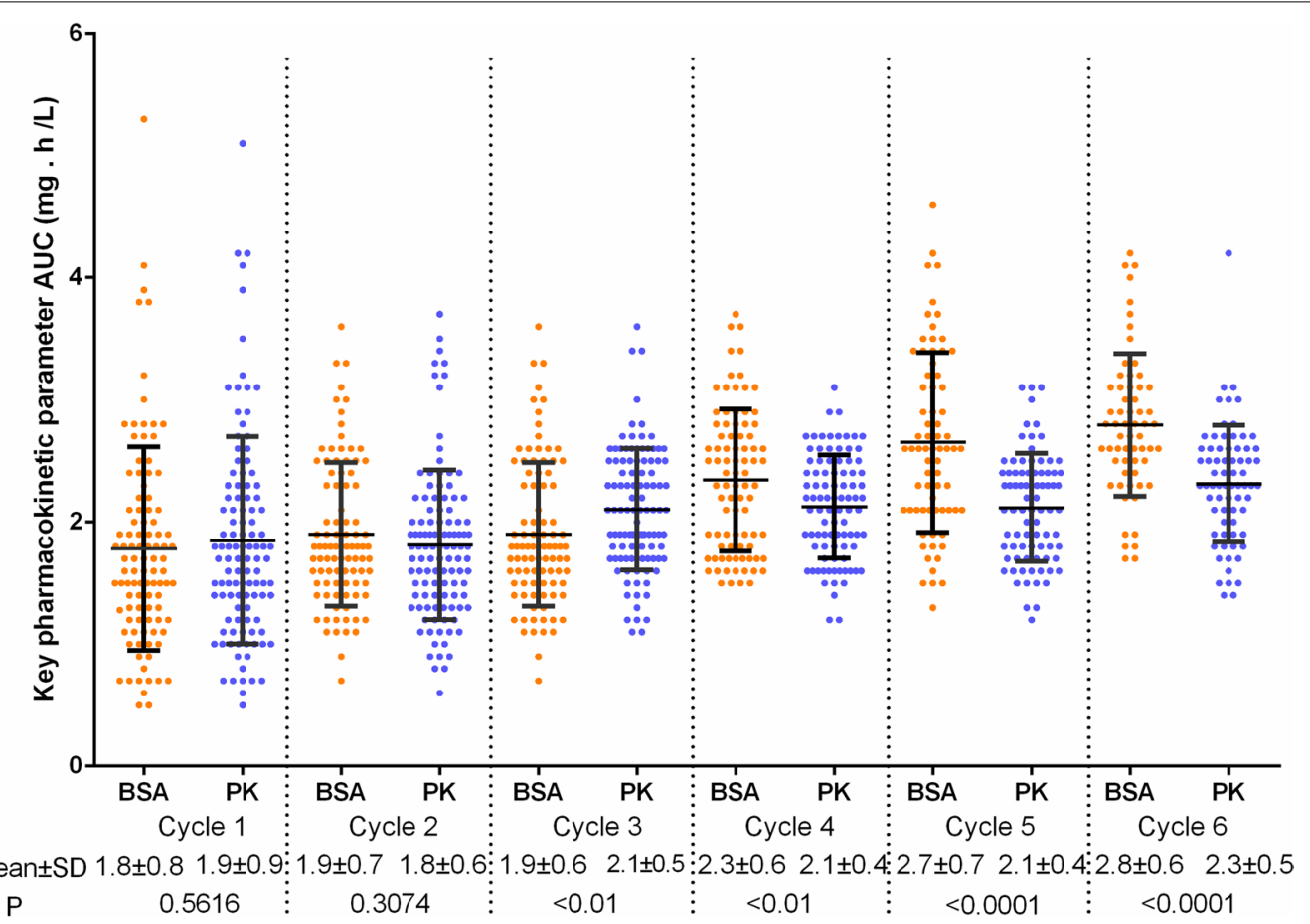

Fig. 1 Comparison of the key PK parameters of AUC of each treatment cycle between BSA and PK groups. From the third treatment cycle, the AUC of paclitaxel in the PK group was significantly lower than that in the BSA group 
Table 2 Proportion of patients within the therapeutic window

\begin{tabular}{lllr}
\hline & $\begin{array}{l}\text { Experimental (PK) } \\
\text { group }\end{array}$ & Control (BSA) group & P value \\
\hline Cycle 1 & $36.70(0.87)$ & $36.00(0.81)$ & 0.9166 \\
Cycle 2 & $50.46(0.62)$ & $41.00(0.66)$ & 0.1705 \\
Cycle 3 & $61.90(0.50)$ & $50.00(0.58)$ & 0.0928 \\
Cycle 4 & $62.64(0.42)$ & $48.24(0.58)$ & 0.0546 \\
Cycle 5 & $71.08(0.43)$ & $36.62(0.72)$ & $<0.0001$ \\
Cycle 6 & $63.89(0.43)$ & $28.33(0.59)$ & $<0.0001$ \\
\hline
\end{tabular}

Data presented as \% (SD) unless otherwise indicated; SD standard derivation of AUC

\section{AUC of DTX amongst patients with different cancer types}

The AUC of paclitaxel amongst patients with lung cancer, breast cancer and gastric cancer under BSA- and PKguided medication was further analysed. In lung cancer and breast cancer, the AUC of cycles 5 and 6 of the PK group was significantly lower than that of the BSA group, but the difference in the AUC of cycles 1-4 between the PK and BSA groups was not significant (Fig. 2). In gastric carcinoma, no significant difference in AUC was observed between PK and BSA groups for cycles 1-6 (Fig. 2).

\section{Incidence of neutropenia}

The incidence rates of neutropenia with grade 3 and above were $29.00 \%$ versus $27.52 \%(P=0.814), 32.00 \%$ versus $32.11 \%(P=0.986)$ and $41.30 \%$ versus $36.19 \%$ $(P=0.467)$ in the control group versus the experimental group in the first, second and third cycles of chemotherapy, respectively. No significant differences were observed. In the fourth, fifth and sixth chemotherapy cycles, the incidence rates were $49.41 \%$ versus $34.07 \%$ $(P=0.039), 56.34 \%$ versus $36.14 \%(P=0.012)$ and $68.33 \%$ versus $38.89 \%(P=0.001)$ in the control group versus the experimental group (Fig. 3 and Table 3). From the fourth cycle, the incidence rate of neutropenia between the control and experimental groups began to show significant differences. The toxicity of the PK group with dosage adjustment was lower than that of the BSA group during the whole chemotherapy cycle. This result indicated that the PK-guided dosage adjustment of DTX could effectively control the toxicity of chemotherapy and reduce its side effects.

\section{Efficacy}

Efficacy is listed in Table 4. After data collection was completed in March 2018, 34 of 100 (34\%) patients in the control group and 40 of 109 patients (37\%) in the experimental group reached the best overall response of PR, 38 of 100 (38\%) patients in the control group and 53 of 109 patients (49\%) in the experimental group achieved the best overall SD response, and 28 of 100 (28\%) patients in the control group and 16 of 109 patients (15\%) in the experimental group achieved the best overall PD response. ORR and DCR were $34 \%$ versus $37 \%$ $(P=0.68)$ and $72 \%$ versus $85 \%(P=0.018)$ in the control group versus the experimental group, respectively. The efficacy of the PK group was significantly higher than that of the control group. The 3-year survival rate of the PK group was significantly higher than that of the BSA group (67.8\% in the PK group vs. $39.0 \%$ in the BSA group, $P=0.034)$, indicating that the PK-guided dosage adjustment of DTX significantly affected the improvement of the survival rate (Fig. 4).

\section{Discussion}

This single-centre, prospective and randomised controlled trial amongst patients who had solid tumours and received DTX-based chemotherapy met its primary endpoint of the population rate within the therapeutic window, the incidence rate of neutropenia and the secondary endpoint of DCR. This study also explored the optimised AUC range for Chinese patients.

Many studies have shown that the data regarding the statistical correlation between BSA and AUC of DTX are insufficient; other factors also affected the clearance for doxorubicin, such as concomitant drugs, infusion duration and sex. In addition, the above studies mainly focused on the solid tumors, not the other types of cancer $[12,13]$. Similar to most chemotherapeutic agents, DTX is administered in accordance with BSA. However, two patients with the same BSA are often encountered clinically, and their efficacy and adverse reactions may be completely different when they receive the same dose of the same chemotherapeutic drug. This difference is probably attributed to the varying PK amongst individuals. Studies have shown that neutropenia is associated with an excessive BSA, a low BMI and a long chemotherapy cycle [14]. Therefore, patients who receive BSA-based dosage may have severe toxic reactions because of high dosage, leading to adverse events. In addition, patients rarely have an opportunity to use high doses to achieve their maximum tolerated dose in a chemotherapy course. Thus, a considerable proportion of patients do not reach the most effective dose of DTX. Remarkable PK differences exist between individuals whose doses are dependent on BSA, and achieving an optimal AUC is difficult [15]. The relationship between the bioavailability of drugs and AUC is observed in patients after the BSA-based treatment was administered, and the difference in the plasma concentration of DTX between individuals is 

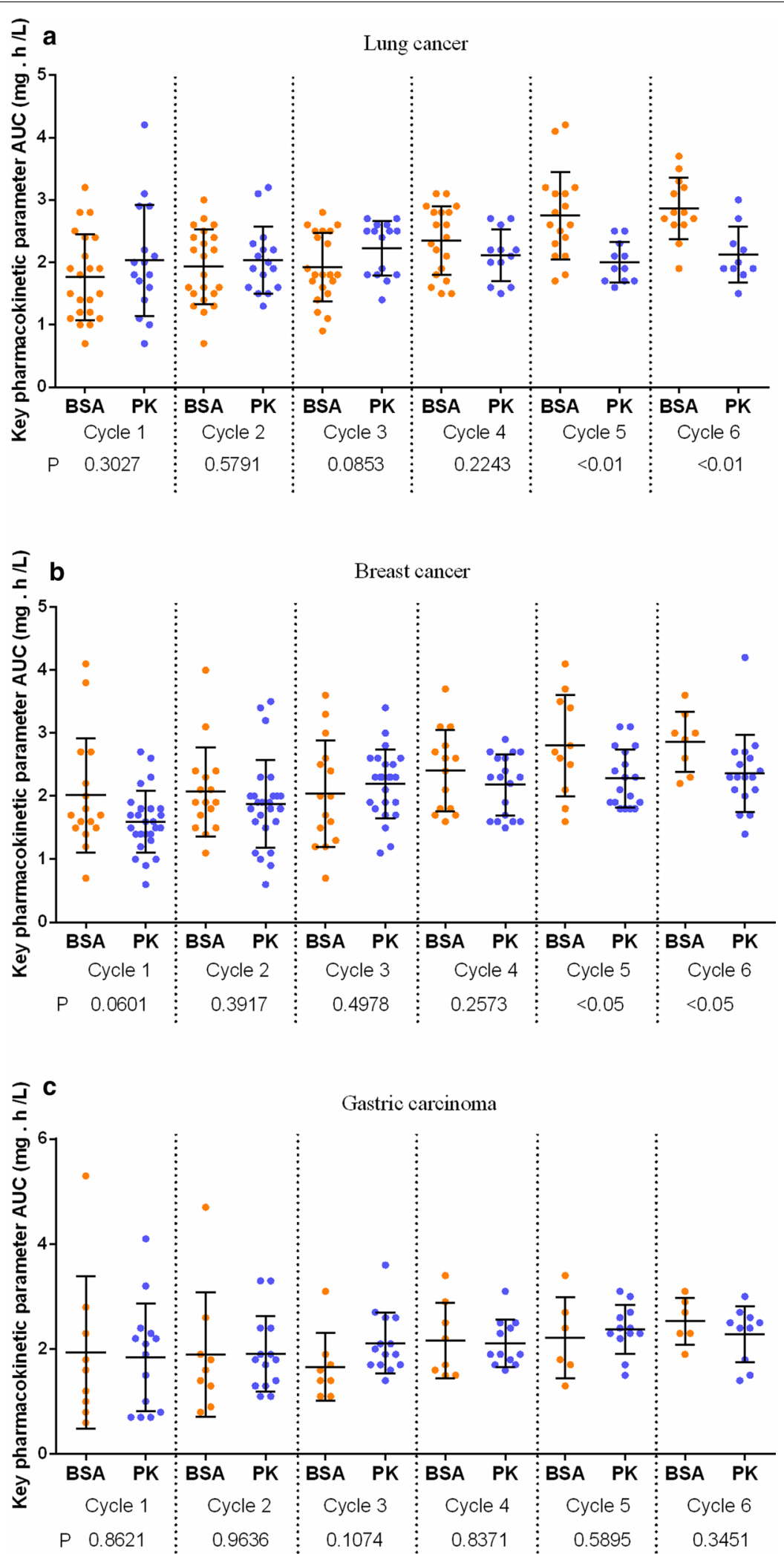

Fig. 2 Comparison of the AUC of each treatment between BSA and PK groups in lung, breast and stomach cancers 
Table 3 Incidence rate of neutropenia with grade 3 and above

\begin{tabular}{llll}
\hline & Experimental (PK) group & Control (BSA) group & $\boldsymbol{P}$ \\
\hline Cycle 1 & $27.52(30 / 109)$ & $29.00(29 / 100)$ & 0.814 \\
Cycle 2 & $32.11(35 / 109)$ & $32.00(32 / 100)$ & 0.986 \\
Cycle 3 & $36.19(38 / 105)$ & $41.30(38 / 92)$ & 0.467 \\
Cycle 4 & $34.07(31 / 91)$ & $49.41(42 / 85)$ & 0.039 \\
Cycle 5 & $36.14(30 / 83)$ & $56.34(40 / 71)$ & 0.012 \\
Cycle 6 & $38.89(28 / 72)$ & $68.33(41 / 60)$ & 0.001 \\
\hline
\end{tabular}

Data presented as \% (No. of grade 3 and above AE/Total patients) unless otherwise indicated. $A E$ adverse effects

Table 4 Efficacy evaluation

\begin{tabular}{lcc}
\hline & PK group $(\boldsymbol{n}=109)$ & BSA group $(\boldsymbol{n}=\mathbf{1 0 0})$ \\
\hline$C R$ & $0(0.00)$ & $0(0.00)$ \\
PR & $40(36.70)$ & $34(34.00)$ \\
SD & $53(48.26)$ & $38(38.00)$ \\
PD & $16(14.68)$ & $28(28.00)$ \\
\hline
\end{tabular}

Data presented as No. (\%) unless otherwise indicated. $C R$ complete recession, $P R$ partial recession, $S D$ stable disease, $P D$ progressed disease

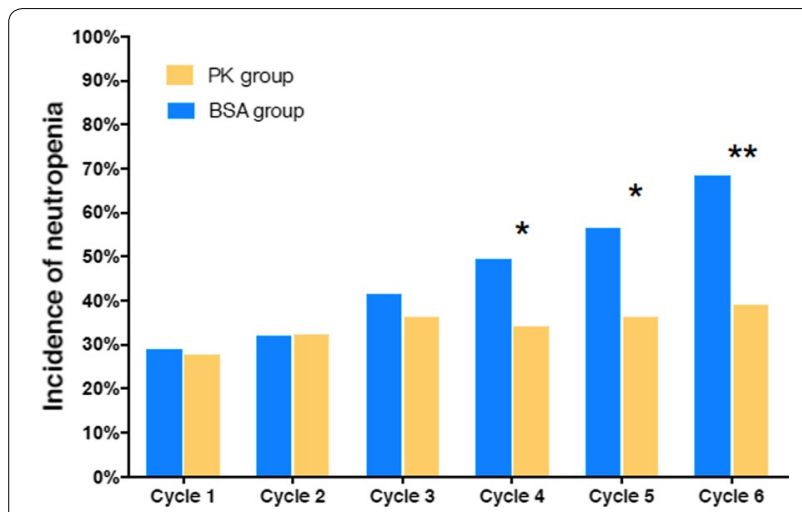

Fig. 3 Incidence of neutropenia amongst the PK and BSA groups. From the fourth treatment cycle, the incidence of grade 3 and above neutropenia in the PK group was significantly lower than that in the BSA group. ${ }^{*}, P<0.05$ compared with the BSA group; ${ }^{*}, P<0.01$ compared with the BSA group

seven times [15]. These differences reflect the variations in the drug clearance rate between individuals and indicate the need for an appropriate method of determining doses.

With the application of reversed phase high-performance liquid chromatography, high-performance liquid chromatography-tandem mass spectrometry and novel nano-enhanced immunoturbidimetric assay in clinical practice, physicians can use PK as a clinical medication guide. The amount of medicine absorbed in blood

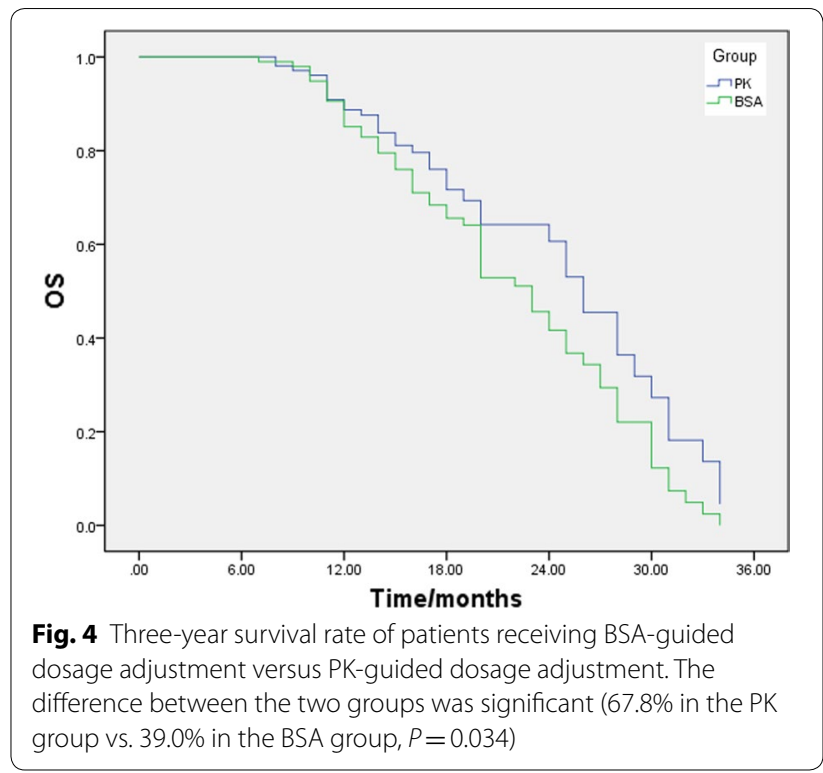

circulation after a single dose can be expressed using AUC, which reflects the relative amount of medicine entering the blood circulation. The range of the DTX therapeutic window is not unified because of the differences in AUC detection and calculation methods and the variety of toxicity criteria amongst research groups. Minami [16], Ozawa [17] and Engels [12] showed that the optimal AUC range of DTX for European and American patients is $2.5-3.7 \mathrm{mg} \mathrm{h} / \mathrm{L}$. However, the optimal AUC range for Asians, especially for the Chinese population, is not well defined. In the present study, the average AUC of DTX was $1.8 \mathrm{mg} \mathrm{h} / \mathrm{L}$, and the AUC of the majority of patients was $1.7-2.5 \mathrm{mg} \mathrm{h} / \mathrm{L}$. Therefore, the therapeutic window in this study was set to $1.7-2.5 \mathrm{mg} \mathrm{h} / \mathrm{L}$. This difference could be related to genetic background, living habits and other factors affecting the PK of DTX. With limited data and sample size, the mechanism could not be explained yet, and this range was preliminary. More data should be collected to validate and modify the feasible target range for Asian patients.

In this study, the patients from the experimental group received PK-guided dosage adjustment. From the second to the sixth cycles, the dosage of the PK group was adjusted in accordance with the AUC of the previous cycle. In the sixth cycle, the AUC in the PK group was 1.4-3.1 $(2.28 \pm 0.43) \mathrm{mg} \mathrm{h} / \mathrm{L}$ and less dispersed compared with that of the BSA group with a range of $1.8-4.2(2.85 \pm 0.59)$. The proportion of the patients in the therapeutic window increased after each cycle of adjustment. After six cycles of adjustment, their proportion reached $63.89 \%$ versus $28.33 \%(\mathrm{P}<0.0001)$ in the experimental group versus the control group. Thus, 
PK-guided dosage adjustment can effectively optimise DTX dosage. The incidence of neutropenia (grade 3 or above) in the experimental group was also significantly lower than that of the control group after three cycles of dosage adjustment. PK-guided dosage adjustment significantly increased the DCR and improved the survival rate of patients. Therefore, PK-guided dosage adjustment based on AUC detection could be a potential therapeutic option to optimise efficacy, reduce toxicity and improve the living quality of patients.

The effects of PK-guided medication on patients with lung cancer, breast cancer and gastric cancer were investigated. At the late stage of chemotherapy for lung cancer and breast cancer, the AUC of PK-guided therapy was significantly lower than that of BSA-guided one. In the whole course of chemotherapy for gastric cancer, the difference in AUC between the PK- and BSA-guided drugs was not significant. Therefore, the PK guidance for DTX was in applicable to all malignant tumours. The value of PK-guided DTX for patients with lung cancer and breast cancer was higher than that for patients with gastric cancer.

This study has several limitations. The proportion of the patients within the therapeutic window and the incidence of neutropenia significantly differed after several cycles of adjustment. However, the first two cycles were not significant, indicating that dosage adjustment should be further optimised. The PK-guided dosage adjustment based on AUC detection did not prolong the patients' life in our study although their quality of life significantly improved. With limited data, the difference in the therapeutic window between Chinese, European and American populations could not be explained. Further studies are needed to answer these questions.

\section{Conclusion}

The PK-guided dosage adjustment of DTX could significantly increase the proportion of patients within the therapeutic window, decrease the incidence of neutropenia and increase the DCR and the 3-year survival rate. The PK-guided dosage adjustment based on the dynamic monitoring of AUC could be a useful method for oncologists to improve individualised treatment options, optimise drug efficacy and reduce drug toxicity.

\section{Abbreviations}

AUC: Area under a curve; BSA: Body surface area; CR: Complete response; DCR: Disease control rate; DTX: Docetaxel; ORR: Objective response rate; PK: Pharmacokinetics; PR: Partial response; SD: Stable disease.

\section{Acknowledgements}

This work was supported by National Natural Science Foundation (Grant No. 81972822) and Maternal and Child Health Project of Jiangsu Province Health Committee (Grant No. F201914).

\section{Author's contributions}

Conception and design of the research: BS and $Y Z$; Performing the clinical experiments: BS, NS and JZ; Interpreted the results: $X Z$ and $H Z$; Prepared figures and tables: BS, GL and JL; Draft manuscript: BS, DY and YZ. All authors read and approved the final manuscript.

\section{Funding}

This work was supported by National Natural Science Foundation (Grant No. 81972822) and Maternal and Child Health Project of Jiangsu Province Health Committee (Grant No. F201914).

\section{Availability of data and materials}

The datasets used and analyzed during the current study are available from the corresponding author on reasonable request.

\section{Ethics approval and consent to participate}

The study protocol and the informed consent procedure were approved by the Ethics Committees of The Affiliated Cancer Hospital of Nanjing Medical University. Written consents were obtained from the subjects before this study.

\section{Consent for publication}

All authors of the manuscript have read and agreed to its content and are accountable for all aspects of the accuracy and integrity of the manuscript.

\section{Competing interests}

The authors declare that they have no competing interests.

\section{Author details}

${ }^{1}$ Department of Medical Oncology, Jiangsu Cancer Hospital \& Jiangsu Institute of Cancer Research \& The Affiliated Cancer Hospital of Nanjing Medical University, No. 42, Baiziting, Nanjing 210009, China. ${ }^{2}$ Key Laboratory of Environmental Medicine Engineering, Ministry of Education, School of Public Health, Southeast University, Nanjing, Jiangsu, China.

Received: 23 December 2019 Accepted: 29 May 2020

Published online: 08 June 2020

\section{References}

1. Herbst RS, Khuri FR. Mode of action of docetaxel—a basis for combination with novel anticancer agents. Cancer Treat Rev. 2003;29:407-15.

2. Shou M, Martinet M, Korzekwa KR, Krausz KW, Gonzalez FJ, Gelboin HV. Role of human cytochrome P450 3A4 and 3A5 in the metabolism of taxotere and its derivatives: enzyme specificity, interindividual distribution and metabolic contribution in human liver. Pharmacogenetics. 1998:8:391-401.

3. Montero A, Fossella F, Hortobagyi G, Valero V. Docetaxel for treatment of solid tumours: a systematic review of clinical data. Lancet Oncol. 2005;6:229-39.

4. Yang YT, Nagai S, Chen BK, Qureshi ZP, Lebby AA, Kessler S, Georgantopoulos P, Raisch DW, Sartor O, Hermanson T, et al. Generic oncology drugs: are they all safe? Lancet Oncol. 2016;17:e493-501.

5. Faqeer NA, Mashni O, Dawoud R, Rumman A, Hanoun E, Nazer L. Comparing the incidence of febrile neutropenia resulting in hospital admission between the branded docetaxel and the generic formulations. J Clin Pharmacol. 2017;57:275-9.

6. Yano R, Konno A, Watanabe K, Tsukamoto H, Kayano Y, Ohnaka H, Goto N, Nakamura T, Masada M. Pharmacoethnicity of docetaxel-induced severe neutropenia: integrated analysis of published phase II and III trials. Int J Clin Oncol. 2013;18:96-104.

7. Nieuweboer AJ, Smid M, de Graan AM, Elbouazzaoui S, de Bruijn P, Eskens FA, Hamberg P, Martens JW, Sparreboom A, de Wit R, et al. Role of genetic variation in docetaxel-induced neutropenia and pharmacokinetics. Pharmacogenomics J. 2016;16:519-24. 
8. Pfeil AM, Vulsteke C, Paridaens R, Dieudonne AS, Pettengell R, Hatse S, Neven P, Lambrechts D, Szucs TD, Schwenkglenks M, Wildiers H. Multivariable regression analysis of febrile neutropenia occurrence in early breast cancer patients receiving chemotherapy assessing patient-related, chemotherapy-related and genetic risk factors. BMC Cancer. 2014;14:201.

9. Baker SD, Li J, ten Tije AJ, Figg WD, Graveland W, Verweij J, Sparreboom A. Relationship of systemic exposure to unbound docetaxel and neutropenia. Clin Pharmacol Ther. 2005;77:43-53.

10. Bruno R, Vivier N, Veyrat-Follet C, Montay G, Rhodes GR. Population pharmacokinetics and pharmacokinetic-pharmacodynamic relationships for docetaxel. Invest New Drugs. 2001;19:163-9.

11. Alexandre J, Rey E, Girre V, Grabar S, Tran A, Montheil V, Rabillon F, Dieras V, Jullien $V$, Herait $P$, et al. Relationship between cytochrome 3A activity, inflammatory status and the risk of docetaxel-induced febrile neutropenia: a prospective study. Ann Oncol. 2007;18:168-72.

12. Engels FK, Loos WJ, van der Bol JM, de Bruijn P, Mathijssen RH, Verweij $J$, Mathot RA. Therapeutic drug monitoring for the individualization of docetaxel dosing: a randomized pharmacokinetic study. Clin Cancer Res. 2011;17:353-62.

13. Rudek MA, Sparreboom A, Garrett-Mayer ES, Armstrong DK, Wolff AC, Verweij J, Baker SD. Factors affecting pharmacokinetic variability following doxorubicin and docetaxel-based therapy. Eur J Cancer. 2004;40:1170-8.
14. Kim WY, Woo SU, Seo JH, Son GS, Lee JB, Bae JW. Toxicities, dose reduction and delay of docetaxel and paclitaxel chemotherapy in breast cancer without distant metastases. J Cancer Res Ther. 2011;7:412-5.

15. Beumer JH, Chu E, Salamone SJ. Body-surface area-based chemotherapy dosing: appropriate in the 21st century? J Clin Oncol. 2012;30:3896-7.

16. Minami H, Ohe Y, Niho S, Goto K, Ohmatsu H, Kubota K, Kakinuma R, Nishiwaki Y, Nokihara H, Sekine I, et al. Comparison of pharmacokinetics and pharmacodynamics of docetaxel and Cisplatin in elderly and nonelderly patients: why is toxicity increased in elderly patients? J Clin Oncol. 2004;22:2901-8.

17. Ozawa K, Minami H, Sato H. Clinical trial simulations for dosage optimization of docetaxel in patients with liver dysfunction, based on a log-binominal regression for febrile neutropenia. Yakugaku Zasshi. 2009;129:749-57.

\section{Publisher's Note}

Springer Nature remains neutral with regard to jurisdictional claims in published maps and institutional affiliations.
Ready to submit your research? Choose BMC and benefit from:

- fast, convenient online submission

- thorough peer review by experienced researchers in your field

- rapid publication on acceptance

- support for research data, including large and complex data types

- gold Open Access which fosters wider collaboration and increased citations

- maximum visibility for your research: over 100M website views per year

At BMC, research is always in progress.

Learn more biomedcentral.com/submissions 